\title{
Publisher Correction: SARS-CoV-2 and HIV-1 - a tale of two vaccines
}

\section{Barton F. Haynes (1)}

Correction to: Nature Reviews Immunology https://doi.org/10.1038/s41577-021-00589-w, published online 16 July 2021.

The sentence "Like SARS-CoV-2-neutralizing antibodies, HIV-1 non-neutralizing antibodies are easily induced by any of a number of HIV-1 Env monomers or open trimerics" should read "Like SARS-CoV-2-neutralizing antibodies, HIV-1 non-neutralizing antibodies are easily induced by any of a number of HIV-1 Env monomers or open trimers". This correction was missed during the proofing process and has now been corrected in the HTML and PDF versions of the article in order to ensure correct scientific terminology.

https://doi.org/10.1038/s41577-021-00604-0 | Published online 4 August 2021

(c) Springer Nature Limited 2021 